\title{
Ideación suicida e insatisfacción con la imagen corporal en jóvenes escolari- zados de la región Altos Sur de Jalisco
}

\section{Suicidal ideation and dissatisfaction with body image in high school students of the region Altos Sur de Jalisco}

\author{
Caldera Montes, Juan Francisco ${ }^{1}$; Reynoso González, Oscar Ulises ${ }^{1, ~}{ }^{*}$; Martín del Campo Ra- \\ yas, Patricia ${ }^{1}$; Gregorio León, Yessica Janeth ${ }^{1}$; Martín Alcalá, Yesenia Anaí ${ }^{1}$ y Arredondo Her- \\ nández, Diana Lady ${ }^{1}$
}

\begin{abstract}
Resumen:
El presente trabajo se planteó como objetivos primordiales, identificar la relación entre la ideación suicida y la insatisfacción con la imagen corporal de jóvenes escolarizados de nivel bachillerato y comparar ambas variables según el sexo de los mismos. La muestra estuvo compuesta por 567 alumnos. Los instrumentos para medir las variables del estudio fueron la Escala de ideación suicida de Beck (SSI) y el Cuestionario de insatisfacción con la imagen corporal (BSQ). Los resultados muestran la presencia de una correlación significativa (positiva y baja) entre dichas variables; que no existen discrepancias en los niveles de ideación suicida conforme el sexo y que sí las hay en el caso de la insatisfacción con la imagen corporal, siendo las mujeres quienes reportan mayores índices. El trabajo concluye afirmando que, la evidencia de que existe relación significativa entre las variables debe tomarse en consideración por parte de los profesionales que atienden este tipo de problemáticas, especialmente, por aquellos expertos de la salud mental que hoy en día abordan la creciente problemática del suicidio en jóvenes y adolescentes.
\end{abstract}

Palabras Clave: ideación suicida, suicidio, insatisfacción con la imagen corporal, adolescente, bachillerato.

\begin{abstract}
:
The main objectives of this study were to identify the relationship between suicidal ideation and dissatisfaction with the body image of high school students and to compare both variables according to sex. The sample consisted of 567 students. The participant answered the Scale of Suicidal Ideation of Beck (SSI) and the Body Image Dissatisfaction Questionnaire (BSQ). The results show the presence of a significant correlation (positive and low) between these variables. There are no discrepancies in the levels of suicidal ideation according to sex, but in the case of dissatisfaction with body image, women are the ones that report the highest rates. The work concludes stating that, the significant relationship between the variables must be taken into consideration by the professionals who deal with this type of problem, especially, by those mental health experts who nowadays attend to the growing problem of suicide in young people and adolescents.
\end{abstract}

Keywords: suicidal ideation, suicide, dissatisfaction whit body shape, teenagers, high school.

\footnotetext{
${ }^{1}$ Universidad de Guadalajara. Centro Universitario de los Altos, Tepatitlán de Morelos, Jalisco, México.

*Correspondencia: ulises.reynoso@academicos.udg.mx
} 
En la actualidad, el suicidio es considerado un problema de salud pública y social que cada día aumenta en términos de prevalencia. En 2012, la OMS publicó un manuscrito exponiendo que el suicidio es la causa de un millón de muertes anuales aproximadamente (citado en Pianowski, Sousa de Oliveira y Nunes, 2015). En dicho informe sobresalen los jóvenes adultos, de entre 15 y 19 años, como un grupo de alto riesgo. México no escapa a estas estadísticas, ya que la tasa de suicidios de la nación ha tenido una tendencia al aumento durante los últimos 50 años: pasando de una tasa de 2.0 durante la década de los setenta (Saltijeral y Terroba, 1987) a una de 5.7 en 2010, año en que el rango de edad de 15 a 24 años presentó las cifras más altas (INEGI, 2011). Incluso hoy en día, el suicidio es la tercera causa de muerte en adolescentes del país (INEGI, 2016).

Como señalan González, Díaz, Ortiz, González y González (2000), a pesar de que el suicidio se ha considerado comúnmente como una expresión de problemas o conflictos mentales subyacentes y prolongados como la depresión, no necesariamente se relaciona con patologías mentales declaradas y su latencia; en el caso de los jóvenes puede ser no prolongada y estar relacionada con problemas propios de dicha etapa de desarrollo. Además, , Rosovsky, Caballero y Gómez (1994) señalan que el suicidio es más común en los jóvenes adultos de 15 a 30 años y gradualmente disminuye al incrementarse la edad; en particular, posterior a los 14 años, la tasa aumenta considerablemente y alcanza el rango más susceptible en el grupo de 20 a 24 años.

En tal sentido, es importante considerar que es un fenómeno en el cual intervienen factores biológicos, sociales y psicológicos (especialmente del desarrollo), y que, debido a su complejidad, el estudio del tema se ha dividido en tres constructos teóricos básicos: la ideación suicida, el intento suicida y el suicidio consumado (Brown, Jeglinc, Henriques y Beck, 2008). Específicamente, la ideación suicida, el antecedente del intento o del suicidio consumado, "corresponde al pensamiento de acabar con la propia existencia" (Córdova y Rosales, 2016), y, según Beck, Kovacs y Weissman (1979), implica el deseo y la planeación de la propia muerte. En ese sentido, la importancia del estudio de la ideación suicida deviene de la utilidad que pudiera tener para prevenir el intento y el suicidio consumado.

Por su parte en la literatura especializada se ha advertido que existen diferentes factores asociados a la ideación suicida como la depresión, sentimientos de desesperanza (Siabato, Forero y Salamanca, 2017; Toro, Grajales y Sarmiento, 2016), abuso sexual, el consumo de alcohol, tabaco o drogas (Pérez, Rivera, Atienzo, Castro, Leyva y Chávez, 2010), ser víctima de situaciones humillantes, falta de apoyo social, experimentación de emociones negativas (Córdova, Rosales y García, 2012), presencia de síntomas psicóticos, ansiosos y problemas afectivos (Mayer, Morales, Victoria y Ulloa, 2016), conductas alimentarias de riesgo y victimización escolar (Sánchez, Villarreal, Musitu y Martínez, 2010). También se ha señalado que la ideación suicida es más frecuente en mujeres que en hombres (Chávez, González, Juárez, Vázquez y Jiménez, 2015).

Al respecto conviene referir que una de las problemáticas que en los últimos años ha sido asociada a la ideación suicida es la denominada "Insatisfacción con la imagen corporal", ya que autores como Piñeros, Molano y López (2010) advierten que tal condi- 
ción aporta a la explicación del riesgo suicida, especialmente en adolescentes. Inclusive, existen estudios en donde se ha señalado que la influencia de los pensamientos negativos sobre el cuerpo se encuentra por encima de los efectos que sobre éstos puede tener la depresión (Muehlenkamp, Claes, Havertaped, \& Plener, 2012).

Específicamente autores como Meza y Pompa (2013) y Vaquero, Alacid, Muyor y López (2013) definen a la Imagen Corporal (IC) como la actitud hacia la apariencia del propio cuerpo, o bien, la forma en cómo uno percibe, imagina, siente y actúa respecto de éste. El termino IC incluye aquellas opiniones, creencias y sentimientos que las personas tienen acerca de su propio cuerpo y de su aspecto físico, asimismo, algunos investigadores la relacionan con la autoestima, que se define como la capacidad de tener confianza y respeto por sí mismo (Giménez, Rivarola y Correché, 2013).

También algunos investigadores sugieren que la IC está formada por cuatro dimensiones diferenciadas: el componente perceptual, que implica la autopercepción del cuerpo, el componente cognitivo, que se refiere a las valoraciones, creencias, constructos personales sobre el propio cuerpo, el componente afectivo, que implica a los sentimientos respecto del mismo, y por último el componente conductual, aquellas acciones o comportamientos que se dan a partir de su percepción (Vaquero, et al., 2013).

Sobre la IC se puede inferir que, al igual que con la ideación suicida, en la etapa de la adolescencia se es más vulnerable a sus efectos. Así, si un joven no se siente satisfecho con su cuerpo es más probable que realice comportamientos que atenten contra la salud física y psicológica (Raich, 2016). Des- afortunadamente, tal condición no es inusitada en el contexto estudiado, ya que como advierten algunas investigaciones realizadas en México, en el país existe una importante preocupación por el aspecto corporal (Cocca, Blanco, Peinado \& Viciana, 2016) y se advierte en algunos casos que la insatisfacción con la imagen del mismo alcanza un $22 \%$ de la población adolescente (Guadarrama, Carri1lo, Márquez, Hernández y Veytia, 2014).

A pesar de tales evidencias, la relación entre la insatisfacción con la imagen corporal e ideación suicida ha sido poco investigada en México, no obstante ello, se pueden consignar como antecedentes del presente trabajo algunos hallazgos en estudios previos que manifiestan una correlación entre ambas variables, por ejemplo: en una investigación efectuada en el estado de México por Guadarrama et al. (2014) se evidenció que el 13\% del total de la población (1054 participantes adolescentes) presentó ideación suicida e insatisfacción con su imagen corporal. Asimismo, indagatorias respecto de la Anorexia o la Bulimia, trastornos alimentarios que se caracterizan por una insatisfacción con la imagen corporal, advierten que estas patologías son factores de riesgo de conducta suicida en escolares (Martínez, Vianchá, Pérez y Avendaño, 2017).

A partir de los referentes anteriores y tomando en cuenta como principal variable de interés la ideación suicida, el presente estudio se planteó como objetivo primordial identificar la posible correlación entre ésta y la insatisfacción con la imagen corporal en jóvenes escolarizados (de nivel bachillerato), además de comparar ambas variables por sexo. Tales propósitos además de generar mayores referentes empíricos sobre el tema, pretenden abonar a la detección de factores de riesgo psicosocial sobre el tema del suicidio y, sobre 
todo, reforzar la idea respecto de la importancia que para los jóvenes hoy en día tiene estar satisfechos con su propio cuerpo.

\section{Tipo de estudio}

\section{Metodología}

El diseño de la investigación fue cuantitativo (dado que el análisis de datos es numérico), no experimental-transversal (ya que no se manipuló ninguna variable y a que se midió en una sola ocasión) y de alcance correlacional (pues se plantea la posible relación entre las variables "ideación suicida" e "insatisfacción con la imagen corporal" y se contrastan diferencias entre las mismas conforme al sexo de los participantes del estudio) (Hernández, Fernández y Baptista, 2014).

\section{Población y muestra}

La población de estudio se conformó por alumnos de un bachillerato tecnológico ubicado en la región Altos Sur de Jalisco, México. Debido a la anuencia y apoyo de la institución, fue posible evaluar a toda la población estudiantil, por lo que participaron un total de 567 alumnos (279 hombres y 288 mujeres) con edades entre los 14 y 20 años $(M=16.29$; $\mathrm{DT}=1.161)$. El plantel educativo en el que se realizó la presente investigación contaba al momento del estudio, con tres carreras técnicas de nivel medio superior, a saber, Técnico Agropecuario, Técnico en Contabilidad y Técnico en Ofimática. Como criterios de inclusión y exclusión, solo participaron alumnos de forma voluntaria y que previamente habían entregado el consentimiento informado firmado por sus padres (en el caso de los menores de edad).

Para una mejor comprensión de la población estudiada, cabe referir que el sistema educativo mexicano, en términos amplios, incluye los niveles educativos de preescolar, primaria, secundaria, bachillerato (general y tecnológico), licenciatura y posgrado (maestría y doctorado). Especificamente, el bachiillerato equivale al último año de ESO y los dos primeros de bachiller del contexto español.

\section{Instrumentos}

Ficha de datos sociodemográficos, el cual incluía las variables de sexo, edad y semestre escolar.

Escala de ideación suicida de Beck (SSI). Tiene un total de 20 ítems los cuales se valoran en una escala de 0 a 2 puntos. Esta escala fue diseñada para evaluar los pensamientos suicidas y su intensidad, además permite conocer si el sujeto ha presentado intentos de suicidio anteriormente (Beck, Kovacs \& Weissman, 1979). Si la puntuación de los reactivos 4 «Deseo de "intentar suicidarse»y 5 «Intento pasivo de suicidarse», es " 0 ”, se omiten las secciones siguientes y se suspende la entrevista, codificando como: "No aplicable". En caso contrario, se sigue aplicando. Dicho instrumento ha sido utilizado en diversos estudios con población mexicana, mostrando altos niveles de confiabilidad y consistencia interna (Córdova, Rosales, Caballero y Rosales, 2007). En el presente trabajo y con la intención de reportar prevalencias y niveles de ideación, se establecieron los rangos siguientes: $\mathrm{Nula}=0, \mathrm{Baja}=1$ a 2, Media $=3 \mathrm{a}$ 6 , Alta $=7$ al1 y Muy Alta $=12+$. El coeficiente de fiabilidad ( $\alpha$ de Cronbach) fue de 915 .

Cuestionario de insatisfacción con la imagen corporal (BSQ) de Cooper, Taylor, Cooper y Fairburn, (1987). Dicho instrumento está conformado por 34 reactivos y tiene como objetivo explorar la autopercepción de 
la imagen corporal e identificar la presencia de insatisfacción con ésta. A través de distintos cuestionamientos sobre aspectos cotidianos, apreciación, hábitos de salud y preocupaciones sobre distintos aspectos del cuerpo permite estimar el nivel de insatisfacción con la forma corporal (p. ej., « ¿Has estado preocupada/o por tu figura que has pensado que debías ponerte a dieta?»). El instrumento fue validado en población mexicana, tanto en hombres (Mendoza, et al., 2010) cómo en mujeres (Vázquez et al., 2011) y presenta un escalamiento tipo Likert de 6 opciones de respuesta, que van de nunca a siempre. El cuestionario ha manifestado índices de confiabilidad adecuados y, aunque algunos trabajos señalan una estructura de 2 factores (Vázquez et al., 2011), para este estudio se tomó el resultado total del cuestionario.

Aunque las versiones por sexo del instrumento tienen ligeras variaciones, en este estudio se optó por utilizar una única versión para ambos segmentos de la población, reportando propiedades psicométricas satisfactorias. Los índices KMO (.975) y la prueba Bartlett $(\mathrm{p}<.000)$ mostraron que el modelo es apropiado, no presenta esfericidad y tiene una buena adecuación muestral (Hair, Anderson, Tatham y Black, 1999). Se extrajeron tres factores bajo el criterio de Kaiser, sin embargo, su interpretación no resultó clara, dando lugar a un modelo unifactorial. El coeficiente de fiabilidad ( $\alpha$ de Cronbach) fue de .976. También en la presente investigación y con el propósito de obtener prevalencias y niveles de insatisfacción, se establecieron los rangos siguientes: Baja $=34$ a 68 , Media $=69$ a 169 y Alta $=170$ a 204.

\section{Procedimiento}

De manera preliminar, se llevó a cabo una entrevista con las autoridades del bachillerato para la presentación, autorización y consentimiento del proyecto de investigación. Una vez autorizado, se visitó, en sus respectivos grupos escolares, a los alumnos que cursaban las carreras (de nivel técnico) antes señaladas y se les pidió que contestaran los instrumentos referidos. El tiempo aproximado para que los participantes respondieran las escalas fue de 25 minutos. Dicho trabajo de campo se llevó a cabo a finales del 2017 y contó en todo momento con el apoyo de profesores y funcionarios de la institución.

\section{Consideraciones éticas}

Durante el proceso de recolección de datos se administró un consentimiento informado tanto a los estudiantes como a los padres de familia cuyos hijos eran menores de edad. En tal documento los investigadores se comprometieron a no divulgar los datos de manera individual, sino globales y sólo con propósitos académicos y científicos. Cabe señalar que durante la aplicación del instrumento se explicaron de forma detalla los objetivos de la investigación tanto a los estudiantes, profesores y directivos de la institución educativa. Por tanto, el estudio está considerado como riesgo mínimo según las normas éticas de la investigación con seres humanos, garantizando los derechos y favoreciendo el bienestar de cada participante. Adicionalmente, tomando a consideración la variable de estudio, se les mencionó a los participantes que si la prueba les generaba algún tipo de incomodidad, podrían negarse a continuar y que el psicólogo escolar estaría disponible para dialogar si así lo quisieran.

\section{Análisis de datos}

Se realizaron análisis descriptivos 
(frecuencias por niveles, medias aritméticas y desviaciones estándar) de la ideación suicida y la insatisfacción con la imagen corporal. Posteriormente se realizaron comparaciones de ambas variables conforme el sexo de los estudiantes, para ello se empleo la prueba de hipótesis denominda U de Mann Whitney debido a que las variables a contrastar no mostraron normalidad en su distribución $(\mathrm{z}=.321$; $\mathrm{p}=0.000$ y $\mathrm{z}=.207 ; \mathrm{p}=0.000$ ). Finalmente $\mathrm{y}$ dado que el proposito primordial de la investigación tenía que ver con la posible correlación entre la ideación suicida y la insatisfacción de la imagen corporal se ejecutó un análisis de correlación mediante la prueba Rho de Spearman. El análisis de los datos se realizó con el programa estadístico SPSS, versión
22.

\section{Resultados}

De forma inicial se calcularon las frecuencias absolutas y relativas de los niveles de ideación suicida e insatisfacción con la imagen corporal de los estudiantes (Tabla 1). Cabe resaltar el hecho de que, bajo la clasificación por niveles, el $44.6 \%$ de alumnos se encontró entre los niveles bajo y muy alto de ideación suicida, mientras que el $27 \%$, se ubicó entre los niveles medios y altos de insatisfacción con la imagen corporal.

También se calcularon las medias y desviaciones estándar de ambas variables. Para su presentación, se muestran los resultados totales y por sexo (Tabla 2).

Tabla 1.

Frecuencias de ideación suicida e insatisfacción de la imagen corporal

\begin{tabular}{lccccc}
\hline \multicolumn{1}{c}{ Variable } & \multicolumn{5}{c}{ Clasificación } \\
\hline \multirow{2}{*}{ Ideación suicida } & Nula & Baja & Media & Alta & Muy alta \\
& 314 & 94 & 46 & 49 & 64 \\
& $55.4 \%$ & $16.6 \%$ & $8.1 \%$ & $8.6 \%$ & $11.3 \%$ \\
Insatisfacción con la & Baja & Media & \multicolumn{2}{c}{ Alta } \\
imagen corporal & & 714 & 147 & 6 \\
& $73.0 \%$ & $25.9 \%$ & & $1.1 \%$ \\
\hline
\end{tabular}

Fuente: elaboración propia.

Tabla 2.

Medias y desviaciones de ideación suicida e insatisfacción con la imagen corporal

\begin{tabular}{lcccccc}
\hline \multirow{2}{*}{ Variable/factor } & \multicolumn{2}{c}{ Total } & \multicolumn{2}{c}{ Mujeres } & \multicolumn{2}{c}{ Hombres } \\
\cline { 2 - 5 } & Me- & D.E. & Media & D.E. & Media & D.E. \\
\hline $\begin{array}{l}\text { Ideación suicida } \\
\text { Insatisfacción corporal con }\end{array}$ & 3.23 & 5.597 & 3.53 & 5.746 & 2.91 & 5.432 \\
\begin{tabular}{l} 
la imagen corporal \\
\hline
\end{tabular} & 60.01 & 31.888 & 69.61 & 36.084 & 50.11 & 23.071 \\
\hline
\end{tabular}

Fuente: elaboración propia. 
Enseguida, con el objetivo de detectar diferencias significativas entre hombres y mujeres en ambas variables, se realizaron pruebas U de Mann Whitney. Los resultados de las mismas se muestran en la Tabla 3.

Como se puede apreciar en la tabla anterior, no se evidenció una diferencia en los
2003).

Finalmente, y dado que el interés primordial del estudio era encontrar posibles correlaciones entre las variables "ideación suicida" e "insatisfacción con la imagen corporal" se utilizó la prueba Rho de Spearman (Tabla 4).

Tabla 3.

Comparación de Ideación suicida e Insatisfacción con la imagen corporal por sexo

\begin{tabular}{lccccc}
\hline \multirow{2}{*}{ Variable/factor } & \multicolumn{2}{c}{ Rango Promedio } & \multirow{2}{*}{ U } & \multirow{2}{*}{ Significancia } & \multirow{2}{*}{ PS } \\
\cline { 2 - 3 } & Hombres & Mujeres & & & \\
\hline Ideación suicida & 272.58 & 295.06 & 36990 & $\mathbf{. 0 7 3}$ & $\mathbf{. 5 4 0}$ \\
$\begin{array}{l}\text { Insatisfacción con la ima- } \\
\text { gen corporal }\end{array}$ & 225.32 & 340.85 & 23804 & $\mathbf{. 0 0 0 *}$ & $\mathbf{. 7 0 4}$ \\
\hline
\end{tabular}

Fuente: elaboración propia

${ }^{*} \mathrm{p}<.01$

niveles ideación suicida entre sexos; por otro lado, se encontraron diferencias significativas en la variable "insatisfacción con la imagen corporal" ( $(p=.000)$, siendo las mujeres quienes obtuvieron puntuaciones superiores. Para dimensionar la magnitud o tamaño del efecto
Como se observa y conforme la prueba de hipótesis empleada, existen relaciones significativas entre las variables analizadas, siendo el nivel de correlación estimado como positivo y bajo en todas. Además de ello, sobresalen las diferencias en el grado de corre-

Tabla 4.

Correlación entre Ideación Suicida e Insatisfacción de la imagen corporal

\begin{tabular}{cccc}
\hline Insatisfacción con la ima- & \multicolumn{3}{c}{ Ideación Suicida } \\
\cline { 2 - 4 } gen corporal & Total & Mujeres & Hombres \\
& $.184^{* *}$ & $.158^{* *}$ & $.246^{* *}$ \\
\hline
\end{tabular}

Fuente: elaboración propia

$* * \mathrm{p}<.01$

se usó la Probabilidad de Superioridad para datos sin normalidad ( $\mathrm{PS}=.704)$. Este valor señala que al seleccionar de forma aleatoria un sujeto de cada grupo, existe un $70.4 \%$ de probabilidad de que el puntaje femenino sea mayor al masculino. De acuerdo a Grissom y Kim (2005), el tamaño de tal efecto podría clasificarse como mediano (Coe y Merino, lación entre hombres y mujeres, siendo menor en el caso de las féminas.

\section{Discusión}

Los resultados del presente estudio exponen prevalencias considerables $(44.6 \%)$ de ideación suicida en los bachilleres abordados destaca que $64(11.35 \%)$ de ellos se ubicó en 
el nivel muy alto-. Dicha condición concuerda con lo ya referido en otras investigaciones (INEGI, 2011; Pianowski, Sousa de Oliveira y Nunes, 2015) que reportan el constante incremento de tal problemática, especialmente en la etapa juvenil. Sobre ello, cabe señalar que, aunque la adolescencia ha sido descrita como una etapa de conflictos de identidad e inestabilidad emocional (Nieto, 2013) la alta prevalencia de ideación suicida no puede atribuirse solamente a dicha condición y muy probablemente ocurra debido a que, en el entorno estudiado, existen condiciones adversas que demeritan aún más la deseable salud mental de sus habitantes. Tal afirmación se sustenta en información reciente que alude alta prevalencia de suicidios consumados en la región geográfica (INEGI, 2011).

Por su parte, el hecho de que al menos 153 jóvenes (27\%) se encuentren en los niveles medio y alto de insatisfacción de la imagen corporal también concuerda con lo reportado en estudios previos como el de Guadarrama et al. (2014). Al respecto, puede esgrimirse que dichas prevalencias pueden deberse a que además de los múltiples cambios experimentados por la adolescencia, tanto físicos como psicológicos (Fernández, González, Contreras y Cuevas, 2015), hoy en día, existe mayor presión sociocultural sobre los modelos de belleza instaurados tanto para los hombres como para las mujeres (Soto et al., 2015).

Que no se adviertan diferencias significativas en la ideación suicida entre los sexos llama la atención, sobre todo porque dichos hallazgos contradicen lo reportado en otros estudios que refieren que son las mujeres quienes obtienen mayores valores en dicha dimensión psicológica (Dion et al., 2015). Ello puede explicarse de manera preliminar señalando que las féminas que cursan las carreras del nivel de estudios abordado, han desarrollado competencias emocionales y cognitivas que las equiparan con el sexo masculino en materia de pretensiones y deseos de vivir. Desde luego y como queda evidente con las contradicciones señaladas, dicho aserto requiere de mayores estudios al respecto.

Con relación a las diferencias entre hombres y mujeres sobre la IC se puede indicar que los datos concuerdan plenamente con indagatorias que han abordado el tema y que específicamente, se pueden explicar aludiendo a lo referido por Trejo, Mollinedo, Araujo, Valdez y Sánchez (2016) en el sentido de que la cultura de la imagen corporal hoy en día es más exigente y suele generar mayores presiones sociales en las mujeres que en los varones.

Respecto de la relación significativa entre la ideación suicida y la insatisfacción con la imagen corporal, conviene decir que también corrobora lo señalado por otros autores como Piñeros, Molano y López (2010) y Brausch y Muehlenkamp (2007). Ello se puede explicar citando a Santrock, (2004) quien ha referido que la búsqueda y obtención de la delgadez se ha convertido en la actualidad en sinónimo de triunfo y éxito, y que, en caso contrario, quienes no logran el control sobre sí mismos y que son rechazados por su contexto, frecuentemente (independientemente de su género) experimentan sentimientos asociados al fracaso, estrés, frustración, tristeza e incluso ideación suicida. Por dichas razones urge implementar medidas de atención y prevención en la zona estudiada, ya que los hallazgos hasta ahora encontrados, llevan a pensar que existen casos extremos de insatisfacción corporal asociada a la ideación suicida.

Cabe destacar que, aunque la investi- 
gación permitió conocer más la problemática consignada, el estudio no está exento de posibles limitaciones, sobre todo en la generalización de los resultados a todos los jóvenes del entorno estudiado. En ese sentido, es recomendable contemplar la inclusión de muestras de jóvenes no escolarizados, así como recopilar información de otros centros educativos del contexto. Asimismo, es necesario indagar sobre otros posibles factores de riesgo suicida en la adolescencia, sobre todo si se toma en cuenta que en el presente estudio las correlaciones entre las variables abordadas, aunque significativas, se catalogan como bajas.

A pesar de ello, se puede concluir afirmando que la evidencia de que existe una relación significativa entre la ideación suicida y la insatisfacción con la imagen corporal debe tomarse en consideración por parte de los profesionales que atienden este tipo de problemáticas; especialmente, por aquellos expertos de la salud mental que hoy en día abordan la problemática, por desgracia cada vez más frecuente, del suicidio en jóvenes y adolescentes.

\section{Referencias}

Beck, A., Kovacs, M. \& Weissman, A. (1979). Assessment of suicidal intention: the Scale for Suicide Ideation. Journal of Consulting and Clinical Psychology. 47(2) 343-352.

Borges, G., Rosovsky, H., Caballero, M. y Gómez, C. (1994). Evolución reciente del suicidio en México: 1970-1991. Anales, Instituto Mexicano de Psiquiatría. 5(9), 15-21. Obtenido de: http:// repositorio.inprf.gob.mx/ handle/123456789/6530

Brausch, A. \& Muehlenkamp, J. (2007). Body image and suicidal ideation in adolescents. Body Image. 4(2), 207-212. doi: 10.1016/ j.bodyim.2007.02.001

Brown, G., Jeglinc, E., Henriques, G. y Beck, A. (2008). Terapia cognitiva, cognición y compor- tamiento suicida. En Thomas E. Ellis (Dir.): Cognición y suicidio. Teoría, investigación y terapia. 51-72, México: Manual Moderno.

Cocca, A., Blanco, J., Peinado, J., \& Viciana, J. (2016). Actual, social and ideal body image in Mexican adolescents and their relation with body dissatisfaction: gender differences. Retos, 30, 189-192. Obtenido de: https://recyt.fecyt.es/ index.php/retos/article/view/50243

Coe, R., y Merino, C. (2003). Magnitud del Efecto: Una guía para investigadores y usuarios. Revista de Psicología de la PUCP, 21(1), 145-177. Obtenido de: revistas.pucp.edu.pe/index.php/ psicologia/article/view/3722/3704

Cooper, P. J., Taylor, M. J., Cooper, Z. \& Fairburn, C. G. (1987). The development and validation of the Body Shape Questionnaire. International Journal of Eating Disorders. 6(4), 485-494. doi:10.1002/1098-108X(198707)6:4<485::AIDEAT2260060405>3.0.CO;2-O

Córdova, M. y Rosales, C. (2016). Ideación suicida: treinta años de investigación en estudiantes universitarios mexicanos. Psicología y Salud. 26 (2), 233-243.

Córdova, M., Rosales, M., Caballero, R. y Rosales, J. (2007) Ideación suicida en jóvenes universitarios: su asociación con diversos aspectos psicosocio-demográficos. Psicología Iberoamericana. 15(2), 17-21. Obtenido de: http:// www.redalyc.org/pdf/1339/133915933003.pdf

Córdova, M., Rosales, J. y García, M. (2012). Ideación suicida y variables asociadas en jóvenes universitarios del estado de Hidalgo (México): Comparación por género. Psicogente. 15(28), 287 301. Obtenido de: http://www.redalyc.org/ pdf/4975/497552361006.pdf

Chávez, A., González, C., Juárez, A., Vázquez, D. y Jiménez, A. (2015). Ideación y tentativas suicidas en estudiantes del nivel medio del estado de Guanajuato, México. Acta universitaria. 25(6), 43-50. doi:10.15174/au.2015.786

Dion, J., Blackburnn, M., Auclairb, J., Laberge, L., Veillette, S., Gaudreault, M... Touchette, É. (2015). Development and aetiology of body dissatisfaction in adolescent boys and girls. International Journal of Adolescence and Youth. 20(2), 151-166. doi:10.1080/02673843.2014.985320

Fernández, J., González, I., Contreras, O. y Cuevas, R. (2015). Relación entre imagen corporal y autoconcepto físico en mujeres adolescentes. Revis- 
ta Latinoamericana De Psicología. 47(1), 2533. doi:10.1016/S0120-0534(15)30003-0

Giménez, P., Rivarola, M. y Correché, M. (2013). Autoestima e Imagen Corporal. Estrategias de intervención psicológica para mejorar el bienestar psicológico en pre- adolescentes en una escuela de la ciudad de San Luis. Argentina. Fundamentos en Humanidades. 27(1), 83-93. Obtenido de: http://www.redalyc.org/ pdf/184/18440029004.pdf

González, S., Díaz, A., Ortiz, S., González, C. y González, J. (2000). Características psicométricas de la Escala de Ideación Suicida de Beck (ISB) en estudiantes universitarios de la ciudad de México. Salud Mental. 23(2), 21-30. Obtenido de: pdf/582/58222304.pdf

Grissom, R., \& Kim, J. (2005). Effect Size for research. A broad practical Approach. Mahwah, New Jersey: Lawrence Erlbaum Associates.

Guadarrama, R., Carrillo S., Márquez O., Hernández J. y Veytia, M. (2014). Insatisfacción corporal e ideación suicida en adolescentes estudiantes del Estado de México. Revista Mexicana de Trastornos Alimentarios. 5(2) 98-106. Obtenido de: http://www.scielo.org.mx/pdf/rmta/v5n2/ v5n2a4.pdf

Hair, J., Anderson, R., Tatham, R. y Black, W. (1999). Análisis multivariante. Madrid: Prentice Hall.

Hernández, R., Fernández, C., y Baptista, P. (2014). Metodología de la investigación. México: McGraw Hill.

Instituto Nacional de Estadística y Geografía [INEGI] (2011). Estadísticas de intentos de suicidio y suicidios. Serie Boletín de Estadísticas, Continuas, Demográficas y Sociales. México: INEGI.

Instituto Nacional de Estadística y Geografía [INEGI] (2016). Estadísticas a propósito del día internacional de la juventud. Comunicación Social, México: INEGI.

Martínez, L., Vianchá, M., Pérez, M. y Avendaño, B. (2017). Asociación entre conducta suicida y síntomas de anorexia y bulimia nerviosa en escolares de Boyacá, Colombia. Acta Colombiana de Psicología, 20(2), 178-188. doi:10.14718/ ACP.2017.20.2.9

Mayer, P., Morales, N., Victoria, G. y Ulloa, R. (2016). Adolescentes con autolesiones e ideación suicida: un grupo con mayor comorbilidad y adversidad psicosocial. Salud Pública de México. 58(3), 335-336. doi:10.21149/spm.v58i3.7893
Mendoza, A., Morales, J., Álvarez, G., Vázquez, R., López, X., y Amaya, A. (2010). Propiedades psicométricas de una versión masculina del Cuestionario de Imagen Corporal (BSQ-V). En A. Hernández (Presidencia) XVIII Congreso Mexicano de Psicología. Sociedad Mexicana de Psicología, Colegio Mexicano de Profesionistas de la Psicología y Universidad Iberoamericana. Ciudad de México, México.

Meza, C. y Pompa, E. (2013). Insatisfacción con la Imagen Corporal en Adolescentes de Monterrey. Daena: International Journal of Good Conscience. 8(1), 32-43. Obtenido de: http:// www.spentamexico.org/v8-n1/A4.8(1)3243.pdf

Muehlenkamp, J., Claes, L., Havertaped, L. \& Plener, L. (2012). International prevalence of adolescent non-suicidal self-injury and deliberate selfharm. Child Adolesc Psychiatry Ment Health. 6 (10). doi: 10.1186/1753-2000-6-10.

Nieto, F (2013). Acercamiento a una Adolescencia Mediatizada. Adolescencia y posmodernidad: Malestares, Vacilaciones y Objetivos. México: Fontamara

Pérez, B., Rivera, L., Atienzo, E., Castro, F., Leyva, A., y Chávez, R. (2010). Prevalencia y factores asociados a la ideación e intento suicida en adolescentes de educación media superior de la República mexicana. Salud Pública de México. 52(4), 324-333. Obtenido de: http:// www.redalyc.org/articulo.oa?id=10617416008

Pianowski, G., Sousa de Oliveira, E. y Nunes, M. (2015). Revisión de ideación, comportamiento suicida y medidas de evaluación psicológicas. Psicología desde el Caribe. 32(1), 81-120. doi:10.14482/psdc.32.1.5872

Piñeros, S., Molano, J. y López, C. (2010). Factores de riesgo de los trastornos de la conducta alimentaria en jóvenes escolarizados en Cundinamarca (Colombia). Revista Colombiana de Psiquiatría. 39(4), 313-328. Obtenido de: http:// www.scielo.org.co/pdf/rcp/v39n2/v39n2a07.pdf

Raich, R. (2016). La tiranía de la imagen. Barcelona: Singlatana.

Saltijeral, M. y Terroba, G. (1987). Epidemiología del suicidio y del parasuicidio en la década de 1971 a 1980 en México. Salud Pública de México. 29, 345-360. Obtenido de: http:// saludpublica.mx/index.php/spm/article/ view/250/241

Sánchez, J., Villarreal, M., Musitu, G., y Martínez. B. 
(2010). Ideación Suicida en Adolescentes: Un Análisis Psicosocial. Psychosocial Intervention. 19(3), 279-287. doi:10.5093/ in2010v19n3a 8

Santrock, J. (2004). Adolescencia. Psicología del desarrollo. España: McGraw Hill.

Siabato, E., Forero, I. y Salamanca, Y. (2017). Asociación entre depresión e ideación suicida en un grupo de adolescentes colombianos. Pensamiento Psicológico, 15(1), 5161. doi:10.11144/Javerianacali.PPSI15-1.ADIS

Soto, M., Marín, B., Aguinaga, I., Guillén, F., Serrano, I., Canga, N.,...Annan, J. (2015). Análisis de la percepción de la imagen corporal que tienen los estudiantes universitarios de Navarra. Nutrición Hospitalaria. 31(5) 2269-2275. doi:10.3305/ nh.2015.31.5.7418

Toro, R., Grajales, F. y Sarmiento, J. (2016). Riesgo suicida según la tríada cognitiva negativa, ideación, desesperanza y depresión. Aquichan. 16 (4), 473-486. doi:10.5294/aqui.2016.16.4.6

Trejo, P., Mollinedo, F., Araujo, R., Valdez, G. y Sánchez M. (2016). Hábitos de actividad física y cánones de imagen corporal en estudiantes universitarios. Revista Cubana de Medicina Integral. 32(1) 72-82. Obtenido de: http:// scielo.sld.cu/pdf/mgi/v32n1/mgi09116.pdf

Vaquero, R., Alacid, F., Muyor, J. y López, P. (2013). Imagen corporal; revisión bibliográfica. Nutrición Hospitalaria. 28(1), 27-35. doi:10.3305/ nh.2013.28.1.6016

Vázquez, R., Galán, J., López, X., Alvarez, G., Mancilla, J., Caballero, A.,...Unikel, C. (2011). Validez del Body Shape Questionnaire (BSQ) en Mujeres Mexicanas. Revista Mexicana de Trastornos Alimentarios. 2(1), 42-52. Obtenido de: Http://www.scielo.org.mx/pdf/rmta/v2n1/ v2n1a5.pdf 